\begin{tabular}{|c|l|}
\hline Title & On the mechanisms for the conversion of ventricular fibrillation to tachycardia by perfusion with ruthenium red \\
\hline Author(s) & Kimura, Hiroyuki; Kawahara, Koichi; Y amauchi, Y oshiko; Miy aki, Jyunichi \\
\hline Citation & $\begin{array}{l}\text { Journal of Electrocardiology, 38(4), 364.370 } \\
\text { https://doi.org/40.1016/.jelectrocard.2005.05.007 }\end{array}$ \\
\hline Issue Date & $2005-10$ \\
\hline Doc URL & http://hdl.handle.net/2115/5930 \\
\hline Type & article (author version) \\
\hline File Information & JE38 4.pdf \\
\hline
\end{tabular}

Instructions for use 


\section{On the mechanisms for the conversion of ventricular fibrillation to tachycardia by perfusion with ruthenium red.}

Hiroyuki Kimura, M. Eng., Koichi Kawahara, Ph. D., Yoshiko Yamauchi, M. Eng., and Jyunichi Miyaki, B. Eng.

Laboratory of Cellular Cybernetics, Graduate School of Information Science and Technology, Hokkaido University, Sapporo 060-0814, JAPAN

Address correspondence to:

Koichi Kawahara, Ph D.

Professor of the Laboratory of Cellular Cybernetics,

Graduate School of Information Science and Technology,

Hokkaido University,

Sapporo 060-0814,

JAPAN

TEL \& FAX: +81-11-706-7591

E-mail: kawahara@cellc.ist.hokudai.ac.jp

Running title: Ruthenium red-induced electrical changes

\section{Acknowledgments:}

This work is supported by a grant-in-aid for scientific research from the Ministry of Education, Science, and Culture of Japan 14658277 and 16300145 to KK. 


\section{Abstract}

We have recently demonstrated that during pacing-induced sustained ventricular fibrillation (VF), perfusion of the heart with either ruthenium red (RR) or Ru 360, blockers of the mitochondrial $\mathrm{Ca}^{2+}$ uniporter, resulted in the reversible conversion of VF to ventricular tachycardia (VT). Here we aimed at elucidating the electrophysiological mechanisms for the RR-induced conversion of VF to VT. The experiments were performed using Langendorff perfused isolated rat hearts in which left ventricular pressure (LVP) and left ventricular intracellular action potential were recorded. Perfusion with either RR or Ru 360 resulted in decreases in the action potential duration $\left(\mathrm{APD}_{90}\right)$, refractory period $(\mathrm{RP})$, and slope of APD restitution curves. These changes were antagonized by co-treatment with Bay K 8644. In addition, perfusion with verapamil produced the decreases in $\mathrm{APD}_{90}, \mathrm{RP}$, and slope of APD restitution curves similar to the RR or Ru 360 perfusion. Such electrophysiological changes may be responsible for the reversible conversion of sustained VF to VT caused by perfusion with RR or Ru 360.

Keywords: Ventricular fibrillation; Ventricular tachycardia; Action potential duration; 
Mitochondria; Ruthenium red; restitution curve 


\section{Introduction}

Ventricular fibrillation (VF) and reentrant ventricular tachycardia (VT) are the major immediate causes of sudden cardiac death [1]. Clinical studies have shown that VF is almost always preceded by VT of variable duration, from a few to many beats [2,3]. Also in the isolated rat heart, a spontaneous transition from VT to VF is frequently observed during reperfusion after global ischemia [4]. We have recently demonstrated that perfusion of isolated rat hearts with ruthenium red (RR) or Ru 360, blockers of $\mathrm{Ca}^{2+}$ uptake by mitochondria, resulted in the reversible conversion of pacing-induced sustained VF to VT, suggesting that changes in mitochondrial $\mathrm{Ca}^{2+}$ uptake were possibly involved in the transition between VT and VF [5].

The RR-induced reversible conversion of sustained VF to VT is antagonized by co-treatment with S(-)-Bay K8644, an activator of L-type $\mathrm{Ca}^{2+}$ channels, suggesting that the inactivation of L-type $\mathrm{Ca}^{2+}$ channels is responsible for the RR-induced effect on the macro-dynamics of hearts [5]. In addition, we have also demonstrated that perfusion with verapamil, a blocker of L-type calcium channels, during sustained VF resulted in the reversible conversion of VF to VT [6].

Rate-dependent alterations of action potential duration (APD) are known to reflect the 
dependence of APD on the preceding diastolic interval (DI), a relationship characterized by electrical restitution [7-9]. Previous experimental and computer simulation studies have suggested that the kinetics of electrical restitution have important implications for the development of ventricular arrhythmias [10-12]. In particular, a steep slope for the restitution relation has been suggested to be crucial to determining whether single spiral waves of electrical activity split into multiple smaller spirals, resulting in the transition from VT to VF $[13,14]$. Flattening of the APD restitution curve with verapamil, diacetyl monoxime [15], and bretylium [16] has been shown to convert VF to a periodic rhythm, or VT in canine or porcine ventricles.

In this study, we have tried to elucidate whether and how perfusion of isolated rat hearts with either $\mathrm{RR}$ or $\mathrm{Ru} 360$, blockers of the mitochondrial $\mathrm{Ca}^{2+}$ uniporter, produced electrophysiological changes contributing to the RR- or Ru 360-induced conversion of sustained VF to VT in isolated rat hearts. 


\section{Materials and methods}

The animal experiments conformed to the "Principles of laboratory animal care" (NIH publication No. 85-23, revised 1996), as well as the "guide for the care and use of laboratory animals”, Hokkaido University School of Medicine.

Preparation of isolated heart

Male Wistar rats (8 weeks old, 240-260 g) were anesthetized with diethylether and administered heparin at $400 \mathrm{U} / \mathrm{kg}$ intravenously. The chest was opened, the aorta cannulated, and the heart excised and immediately placed in ice-cold Krebs-Henseleit bicarbonate (KHB) buffer. The heart was then connected to the Langendorff apparatus and perfused through the aorta in a non-recirculating constant pressure mode.

A water-filled elastic balloon was inserted into the left ventricle via the left atrium. The left ventricular pressure (LVP) was monitored by a pressure transducer (DT-XXED, Ohmeda, Madison, WI) connected to the balloon, and was continuously monitored and recorded. The initial value of the end diastolic pressure (EDP) was set to 7-10 mmHg by adjusting the volume of the balloon. The intracellular action potentials (AP) of the left ventricular (LV) muscles 
were recorded with machine-pulled glass capillary electrodes filled with $3 \mathrm{M} \mathrm{KCl}$. To avoid motion artifacts caused by rhythmic contractions of LV muscles in the AP recordings, purpose-built suction and floating electrodes [17] were used for recording APs.

\section{Standard restitution protocol}

Hearts were perfused for 20 min with KHB solution (equilibrium period).

Enamel-insulated copper wires ( diameter, $70 \mu \mathrm{m}$ ) were inserted into the left ventricular

muscle. The left ventricle was then electrically stimulated with these electrodes at an intensity

equal to of 3 times the threshold for electrical activation. The dependence of action potential duration (APD) on the preceding diastolic interval (DI) was determined with the use of a standard S1-S2 protocol [18]. Single test pulses (S2) were delivered after every $20^{\text {th }}$ basic pulse (S1) at a basic cycle length (BCL) of 200 ms. This BCL was used because it was the shortest cycle length at which no alternation of APD occurred in the rat hearts used in this experiment. The S1-S2 coupling interval was progressively shortened in steps of 5-10 ms starting from 80 ms until the premature pulse was blocked. The point at which the premature pulse was blocked was defined as the refractory period (RP). After that, the S1-S2 interval 
was increased from $90 \mathrm{~ms}$ in steps of $10 \mathrm{~ms}$, and the diastolic interval (DI)- APD at 90\% repolarization $\left(\mathrm{APD}_{90}\right)$ relationship was measured.

Drugs used

RR, verapamil, and S(-)-Bay K8644 (Bay K) were obtained from Sigma (St. Louis, MO).

Ru360 was obtained from Calbiochem (La Jolla, CA). The other chemicals were from Wako

Chem (Tokyo, Japan).

Statistics

The data are expressed as the mean \pm S.D. Comparisons were performed using the one-way analysis of variance (ANOVA) followed by a paired t-Test. A $P$ value of less than 0.05 or 0.01 was considered statistically significant. 


\section{Results}

We first investigated whether and how perfusion of isolated hearts with $5 \mu \mathrm{M}$

ruthenium red (RR), a blocker of mitochondrial $\mathrm{Ca}^{2+}$ uptake, changed the action potential duration (APD) of left ventricular muscles, left ventricular developed pressure (LVDP), and refractory period (RP). RR perfusion resulted in a significant shortening of APD at 90\% repolarization $\left(\mathrm{APD}_{90}\right)$, and in a significant decrease in LVDP (Fig. 1A2 \& Fig. 2A) as compared with values before the onset of the perfusion (control) (Fig. 1A1 \& Fig. 2A). The RR-induced effect on the $\mathrm{APD}_{90}$ and LVDP was almost reversed 5 min after the washout (WO) of RR (Fig.1 A3 \& Fig. 2A). We then investigated the RR-induced changes in the RP. Before the start of the perfusion (control), successive stimuli (S1 \& S2) with a 50 ms interval induced AP (Fig. 1B1, left), but those with a $40 \mathrm{~ms}$ interval did not (Fig. 1B1, right). In contrast, during RR perfusion, successive stimuli with a $40 \mathrm{~ms}$ interval at the same stimulus intensity induced the AP (Fig. 1B2, right), indicating that the perfusion shortened the RP. After the washout of RR, the stimuli with a 40 ms interval did not induce AP (Fig. 1B3, right). A statistical analysis revealed that RR perfusion significantly decreased the RP reversibly (Fig. 2B). 
We then investigated the RR-induced changes in the APD restitution curve: that is, the relationship between $\mathrm{APD}_{90}$ and diastolic intervals (DI) (Fig. 3). $\quad$ Perfusion with $5 \mu \mathrm{M}$ RR resulted in a flattening of the APD restitution curve (Fig. 3A2) as compared with that before the start of perfusion (control) (Fig. 3A1). It also significantly decreased the maximum slope of the APD restitution curve, reversibly (Fig.3B).

To confirm that the RR-induced changes in the $\mathrm{APD}_{90}$, LVDP, RP and maximum slope of the APD restitution curve were caused by the RR-induced inhibition of $\mathrm{Ca}^{2+}$ uptake by mitochondria, we perfused the heart with $\mathrm{Ru} 360$, a specific blocker of the mitochondrial $\mathrm{Ca}^{2+}$ uniporter in cardiac myocytes [19,20]. $\mathrm{Ru} 360(10 \mu \mathrm{M})$ perfusion significantly reduced the $\mathrm{APD}_{90}$, LVDP (Fig. 4A), and RP (Fig.4B), reversibly. Ru 360 perfusion also decreased the maximum slope of the APD restitution curve (Fig. 4C2) as compared with that before the onset of $\mathrm{Ru} 360$ perfusion (Fig. 4C1). In this heart, the maximum slope showed a rebound-like marked increase 5 min after the washout of Ru 360 (Fig.4 C3), and then gradually returned to that before Ru 360 perfusion.

We have recently demonstrated that the RR-induced reversible conversion of sustained VF to VT is antagonized by co-treatment with S(-)-Bay K8644 (Bay K), an activator of L-type 
$\mathrm{Ca}^{2+}$ channels, suggesting that the inactivation of L-type $\mathrm{Ca}^{2+}$ channels is responsible for the RR-induced effect on the macro-dynamics of hearts [5]. Therefore, we next investigated whether the RR-induced electrophysiological changes observed in Figs. 1 and 2 were also antagonized by co-treatment with Bay K. The RR (5 $\mu$ M)-induced decrease in the APD $\mathrm{A}_{90}$, LVDP and RP were significantly antagonized by co-treatment with $10 \mu \mathrm{M}$ Bay K (Fig. 5A and 5B). In addition, co-treatment with RR and Bay $\mathrm{K}$ did not change the APD restitution curve: that is, the co-treatment did not flatten the restitution curve (Fig. 5C). These results suggested that the inactivation of L-type $\mathrm{Ca}^{2+}$ channels is responsible for the RR-induced effect on the electrophysiological changes such as the decrease in $\mathrm{APD}_{90}$. In fact, perfusion of hearts with verapamil (600 nM), an antagonist of L-type $\mathrm{Ca}^{2+}$ channels, resulted in a decrease in the $\mathrm{APD}_{90}$, LVDP and RP (Fig. 6A and 6B). Verapamil treatment also flattened the maximum slope of the APD restitution curve (Fig. 6C2) as compared with that before the onset of verapamil perfusion (Fig. 6C1), reversibly. 


\section{Discussion}

The present study demonstrated that perfusion of isolated rat hearts with either ruthenium

red (RR) or $\mathrm{Ru} 360$, blockers of $\mathrm{Ca}^{2+}$ uptake by mitochondria, resulted in a shortening of the action potential duration at $90 \%$ repolarization $\left(\mathrm{APD}_{90}\right)$, in a decrease in the left ventricular developed pressure (LVDP), and in the flattening of the maximum slope of the APD restitution curve.

Previous studies have shown that RR not only inhibits $\mathrm{Ca}^{2+}$ uptake by mitochondria but also $\mathrm{Ca}^{2+}$ release channels of SR [21], and sarcolemmal voltage dependent $\mathrm{Ca}^{2+}$ channels [22]. Thus, the question arises as to whether RR-induced reversible electrophysiological changes in the APD, RP, and APD restitution curve were caused by RR-induced pharmacological effects other than the inhibition of $\mathrm{Ca}^{2+}$ uptake by mitochondria. However, perfusion of hearts with $\mathrm{Ru} 360$, a specific blocker of the mitochondrial $\mathrm{Ca}^{2+}$ uniporter $[19,20]$, resulted in almost the same electrophysiological changes as those induced by perfusion with RR (Figs. 4), suggesting that the RR-induced shortening of APD, and decreases in the LVDP, RP, and maximum slope of APD restitution curve, were caused by the inhibition of $\mathrm{Ca}^{2+}$ uptake by mitochondria.

In this study, perfusion of isolated hearts with either RR or Ru 360 resulted in a decrease 
in $\mathrm{APD}_{90}$, $\mathrm{LVDP}$, and RP. The question arises as to what mechanisms are involved in the link between $\mathrm{RR}$ - or $\mathrm{Ru}$ 360-induced inhibition of $\mathrm{Ca}^{2+}$ uptake by mitochondria and such electrophysiological changes. The exact mechanisms are currently unclear, but one possibility is that the local $\mathrm{Ca}^{2+}$ rise in the micro-domains surrounding the mouth of L-type $\mathrm{Ca}^{2+}$ channels, SR and mitochondria caused by the $\mathrm{RR}$ - or $\mathrm{Ru} 360$-induced reduction of $\mathrm{Ca}^{2+}$ uptake by mitochondria would result in the reduced activity of sarcolemmal L-type $\mathrm{Ca}^{2+}$ channels. In support of this idea, a recent study has revealed that the inhibition of mitochondrial $\mathrm{Ca}^{2+}$ uptake by treatment with $\mathrm{RR}$ or $\mathrm{Ru} 360$ results in a reduction in the activity of L-type $\mathrm{Ca}^{2+}$ channels in rat hearts [19]. It is well known that the activity of L-type $\mathrm{Ca}^{2+}$ channels is negatively regulated by an increase in intracellular $\mathrm{Ca}^{2+}\left(\mathrm{Ca}^{2+}\right.$-dependent inactivation of $\mathrm{L}$-type $\mathrm{Ca}^{2+}$ channels) [23]. In addition, RR-induced electrophysiological changes were almost antagonized by co-treatment with Bay $\mathrm{K}$, an activator of L-type $\mathrm{Ca}^{2+}$ channels (Fig. 5). Perfusion with verapamil, an antagonist of L-type $\mathrm{Ca}^{2+}$ channels, resulted in a decrease in the $\mathrm{APD}_{90}$, LVDP and RP (Fig. 6A and B); the effects were almost the same as those induced by RR or Ru 360. All these findings have led to the suggestion that the inhibition of mitochondrial $\mathrm{Ca}^{2+}$ uptake with $\mathrm{RR}$ or $\mathrm{Ru} 360$ decreased the $\mathrm{APD}_{90}$, $\mathrm{RP}$, and slope of the APD restitution curve 
via RR- or Ru 360-induced inactivation of L-type $\mathrm{Ca}^{2+}$ channels.

Previous experimental and computer simulation studies have suggested that the kinetics

of electrical restitution have important implications for the development of ventricular arrhythmias [7-9]. In particular, a steep slope for the restitution relation has been suggested to be a crucial factor in determining whether single spiral waves of electrical activity split into multiple smaller spirals, resulting in the transition from VT to VF [13,14]. In this study, perfusion of isolated hearts with RR or Ru 360 flattened the slope of the APD restitution curve (Fig. 3 \& Fig. 4). The question arises as to what mechanisms are involved in this effect on the APD restitution curve. A previous study has revealed that flattening of the APD restitution curve with verapamil [15] has been shown to convert VF to VT. In addition, perfusion of isolated rat hearts with verapamil flattened the APD restitution curve (Fig. 6). We have also demonstrated that verapamil perfusion converts the pacing-induced sustained VF to VT, reversibly [6]. All these findings have suggested that the RR- or Ru 360-induced change in the APD restitution curve was caused by the inactivation of L-type $\mathrm{Ca}^{2+}$ channels.

In summary, the present study has suggested that the RR- or Ru 360-induced decrease in the APD, RP, and slope of the APD restitution curve was responsible for our previous finding 
that perfusion of isolated rat hearts with either $\mathrm{RR}$ or $\mathrm{Ru} 360$ reversibly converts the pacing-induced sustained VF to VT [5].

Clinical Implications:

Ventricular tachycardia (VT) is considered to be the most common precursor of ventricular fibrillation (VF) and sudden cardiac death. However, the mechanisms underlying the transition from VT to VF remain unclear despite more than a century of study. This study demonstrated the electrophysiological changes of left ventricular muscles caused by the changes in mitochondrial $\mathrm{Ca}^{2+}$ uptake in the transition between VT and VF. Thus, the present results would contribute to the novel therapeutic ideas for the prevention of hearts from the occurrence of lethal arrhythmia (VF) by regulating the function of mitochondria. 


\section{References}

1. Myerburg RJ, Castellanos A: Cardiac arrest and cardiac death. p. 742-779. In Braunwald E (ed): Heart Disease: a textbook of cardiovascular medicine. Saunders, Philadelphia, 1997

2. Nikolic G, Bishop, RL, Singh JB: Sudden cardiac death recorded during Holter monitoring. Circulation 66:218, 1982

3. Pratt CM, Francis MJ, Luck JC, Wyndkham CR, Miller RR, Quinones MA: Analysis of ambulatory electrocardiograms in 15 patients during spontaneous ventricular fibrillation with special reference to preceding arrhythmic events. J Am Coll Cardiol 2:789, 1983

4. Kawahara K, Takase M, Yamauchi Y: Increased vulnerability to ischemia/reperfusion-induced ventricular tachyarrhythmias by pre-ischemic inhibition of nitric oxide synthase in isolated rat hearts. Cardiovasc Pathol 12:49, 2003

5. Kawahara K, Takase M, Yamauchi Y: Ruthenium red-induced transition from ventricular fibrillation to tachycardia in isolated rat hearts: possible involvement of changes in mitochondrial calcium uptake. Cardiovasc Pathol 12:311, 2003

6. Kawahara K, Takase M, Yamauchi Y, Kimura H: Spectral and correlation analyses of the 
verapamil-induced conversion of ventricular fibrillation to tachycardia in isolated rat hearts. J Electrocardiol 37:89, 2004

7. Bass BG: Restitution of the action potential in cat papillary muscle. Am J Physiol 240:1717, 1981

8. Boyett MR, Jewell BR: A study of the factors responsible for the rate-dependebt shortening of the action potential in mammalian muscle. J Physiol (Lond.) 285:359, 1978

9. Boyett MR, Jewell BR: Analysis of the effects of change in rate and rhythm upon the electrical activity in the heart. Prog Biophys Mol Biol 36:1, 1980

10. Kobayashi Y, Peters W, Khan SS, Mandel WJ, Karagueuzian HS: Cellular mechanisms of differential action potential duration restitution in canine ventricular muscle cells during single versus double premature stimuli. Circulation 86:955, 1992

11. Nakaya Y, Varro A, Elharrar V, Surawicz B: Effect of altered repolarization course induced by antiarrhythmic drugs and constant current pulses on duration of premature action potentials in canine cardiac Purkinje fibers. J Cardiovasc Pharmacol 14:908, 1989

12. Saitoh H, Bailey JC, Surawicz B: Action potential duration alternans in dog Purkinje and ventricular muscle fibers. Further evidence in support of two different mechanisms. 
Circulation 80:1421, 1989

13. Chen PS, Karagueuzian HS, Weiss JN, Garfinkel A: Spirals, chaos, and new mechanisms of wave propagation. Pacing Clin Electrophysiol 20:414, 1997

14. Karma A: Electrical alternans and spiral wave breakup in cardiac tissue. Chaos 4:461, 1994

15. Ricco ML, Koller ML, Gilmour RFJ: Electrical restitution and spatiotemporal organization during ventricular fibrillation. Circ Res 84:955, 1999

16. Garfinkel A, Kim YH, Voroshilovsky O, Qu Z, Kil JR, Lee MH, Karagueuzian HS, Weiss JN, Chen PS: Preventing ventricular fibrillation by flattening cardiac restitution. Proc Natl Acad Sci USA 97:6061, 2000

17. Franz MR: Method and theory of monophasic action potential recording. Prog Cardiovasc Diseas 38:347, 1991

18. Koller ML, Ricco ML, Gilmour RF: Dynamic restitution of action potential duration during electrical alternans and ventricular fibrillation. Am J Physiol 275:H1635, 1998

19. Sánchez JA, García MC, Sharma VK, Young KC, Matlib MA, Sheu S-S: Mitochondria regulate inactivation of L-type $\mathrm{Ca}^{2+}$ channels in rat heart. J physiol 536.2:387, 2001 
20. Matlib MA, Zhou Z, Knight S, Ahmed S, Choi KM, Krause-Bauer J, Phillips R, Altschuld R, Katsube Y, Sperelakis N, Bers DM: Oxygen-bridged dinuclear ruthenium amine complex specifically inhibits $\mathrm{Ca}^{2+}$ uptake into mitochondria in vitro and in situ in single cardiac myocytes. J Biol Chem 273:10223, 1998

21. Meissner G, Henderson JS: Rapid calcium release from cardiac sarcoplasmic reticulum vescicles is dependent on $\mathrm{Ca}^{2+}$ and is modulated by $\mathrm{Mg}^{2+}$, adenine nucleotide, and calmodulin. J Biol Chem 262:3065, 1987

22. Vassilev PM, Kanazirska MP, Charamella LJ, Dimitrov NV, Tien HT: Changes in calcium channel activity in membranes from cis-diammine-dichloroplatinum (II)-resistant and -sensitive L1210 cells. Cancer Res 47:519, 1987

23. Anderson ME: $\mathrm{Ca}^{2+}$-dependent regulation of cardiac L-type $\mathrm{Ca}^{2+}$ channels: is a unifying mechanism at hand? J Mol Cell Cardiol 33:639, 2001 


\section{Figure Legends}

Fig. 1

Ruthenium red-induced changes in action potential duration (APD), left ventricular pressure (LVP), and refractory period (RP). Perfusion of isolated hearts with $5 \mu \mathrm{M}$ ruthenium red (RR) reduced the APD and LVP, reversibly. Figures A1, A2, and A3 show APs of the left ventricular muscle and LVP before the start of RR perfusion (A1), 5 min after RR perfusion, and 5min after the onset of the washout of RR (A3), respectively. Figures B1, B2, and B3 show the evoked APs to measure RP before perfusion (B1), 5 min after the start of RR perfusion (B2), and 5 min after the washout of RR (B3), respectively. Diagonal arrows indicate the APs evoked by the stimulus S2. Abbreviations: AP, action potential; APD, action potential duration; LVP, left ventricular pressure.

Fig. 2

Ruthenium red-induced decrease in action potential duration (APD), left ventricular 
developed pressure (LVDP), and refractory period (RP). Perfusion with $5 \mu$ M ruthenium red (RR) resulted in a significant decrease in $\mathrm{APD}_{90}$ and LVDP, reversibly. The perfusion also decreased the RP significantly. Data are expressed as the mean+SD $(n=10) . \quad{ }^{*} \mathrm{p}<0.01$. Abbreviation: LVDP, left ventricular developed pressure. Other abbreviations are the same as those in Fig. 1.

Fig. 3

Changes in the restitution curve of action potential duration (APD) caused by perfusion with ruthenium red (RR). Figures A1, A2, and A3 show the APD restitution curves before perfusion (A1), 5 min after (A2) the onset of RR (5 $\mu \mathrm{M})$ perfusion, and 5 min after the washout of RR (A3), respectively. Data points are indicated by dots, and the bold curve indicates the quadratic curve fitted to data points by the least square methods. Figure B shows the RR-induced significant decrease in the maximum slope of the APD restitution curve. Data are expressed as the mean+SD $(n=6) . \quad * \mathrm{p}<0.05 . \quad$ Abbreviation: DI, diastolic interval. Other abbreviations are the same as those in Figs. 1 and 2. 
Fig. 4

Changes in action potential duration (APD), left ventricular developed pressure (LVDP),

refractory period (RP), and the APD restitution curve caused by perfusion with Ru 360 .

Perfusion with $10 \mu \mathrm{M} \mathrm{Ru} 360$, a specific inhibitor of the uptake of $\mathrm{Ca}^{2+}$ by mitochondria, resulted in a significant decrease in $\mathrm{APD}_{90}$, $\mathrm{LVDP}$, and $\mathrm{RP}$, reversibly. $\mathrm{Ru} 360$ perfusion also decreased the maximum slope of the APD restitution curve. Data are expressed as the mean+SD $(n=4) . \quad * \mathrm{p}<0.05$.

Fig. 5

Ruthenium red (RR)-induced electrophysiological changes are antagonized by co-treatment with an activator of L-type $\mathrm{Ca}^{2+}$ channels. Co-treatment of isolated rat hearts with $5 \mu$ M RR and $10 \mu$ M S(-)-Bay K8644, an activator of L-type $\mathrm{Ca}^{2+}$ channels, did not produce significant changes in action potential duration (APD), left ventricular developed pressure (LVDP), and refractory period (RP). The co-treatment also did not result in the any appreciable changes in the APD restitution curve. Data are expressed as the mean+SD $(n=4)$. * $\mathrm{p}<0.05 . \quad$ Abbreviation: Bay K, S(-)-Bay K8644. 
Fig. 6

Perfusion with an antagonist of L-type $\mathrm{Ca}^{2+}$ channels produced similar electrophysiological changes to those evoked by treatment with ruthenium red (RR). Isolated hearts were perfused with $600 \mathrm{nM}$ verapamil, an antagonist of L-type $\mathrm{Ca}^{2+}$ channels. The perfusion resulted in a decrease in the action potential duration at $90 \%$ repolarization ( $\mathrm{APD}_{90}$ ), left ventricular developed pressure (LVDP), refractory period (RP), and maximum slope of the APD restitution curve, reversibly. Data are expressed as the mean+SD (n=4). $\quad * p<0.05$. 
A1
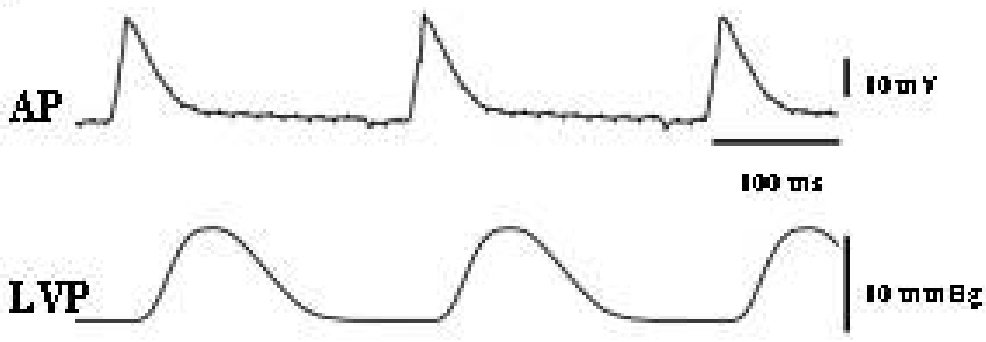

A2

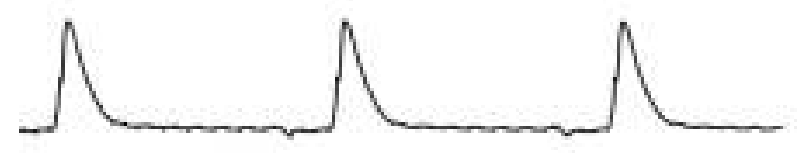

A3

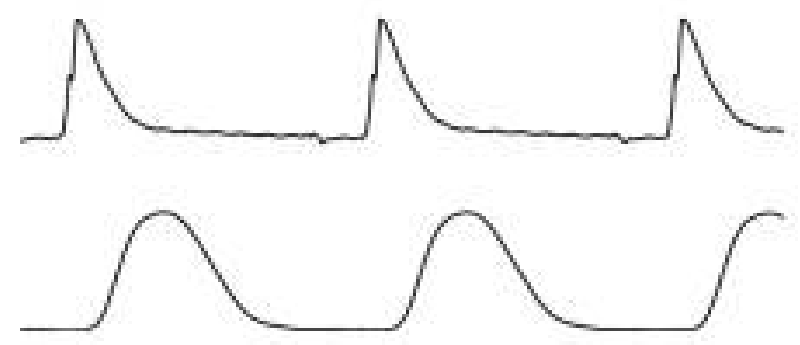

B1

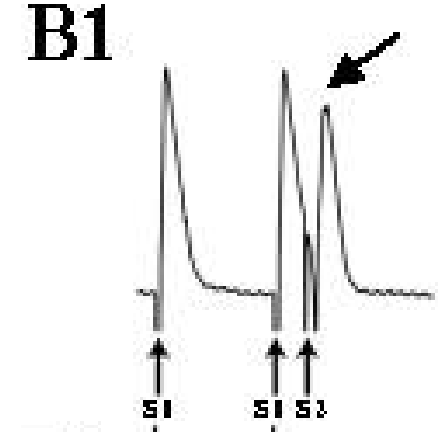

B2

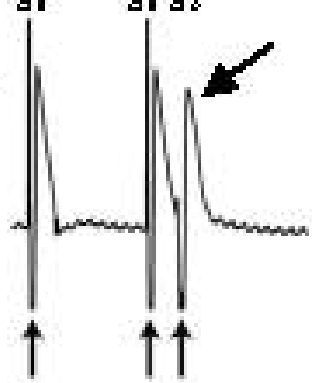

B3

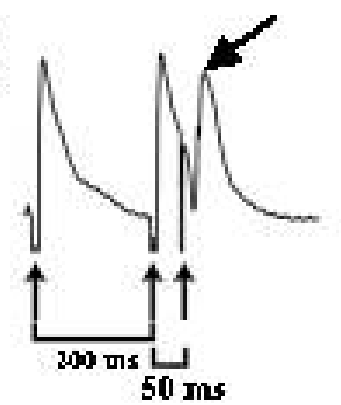

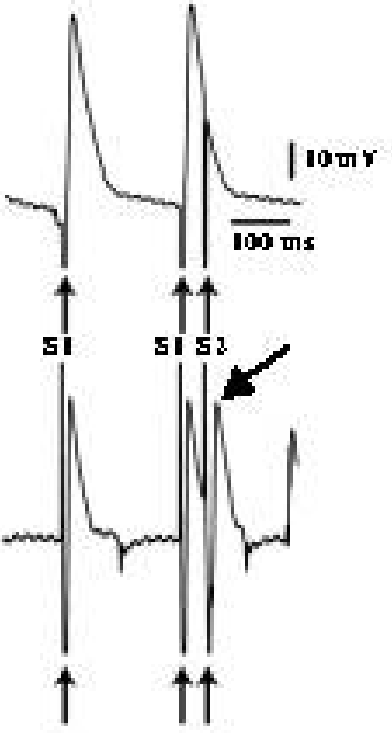

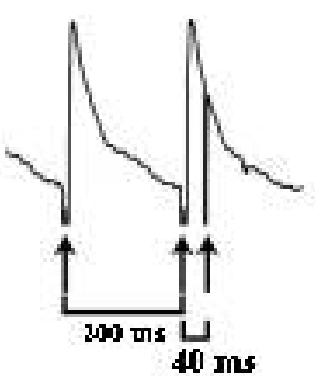


A
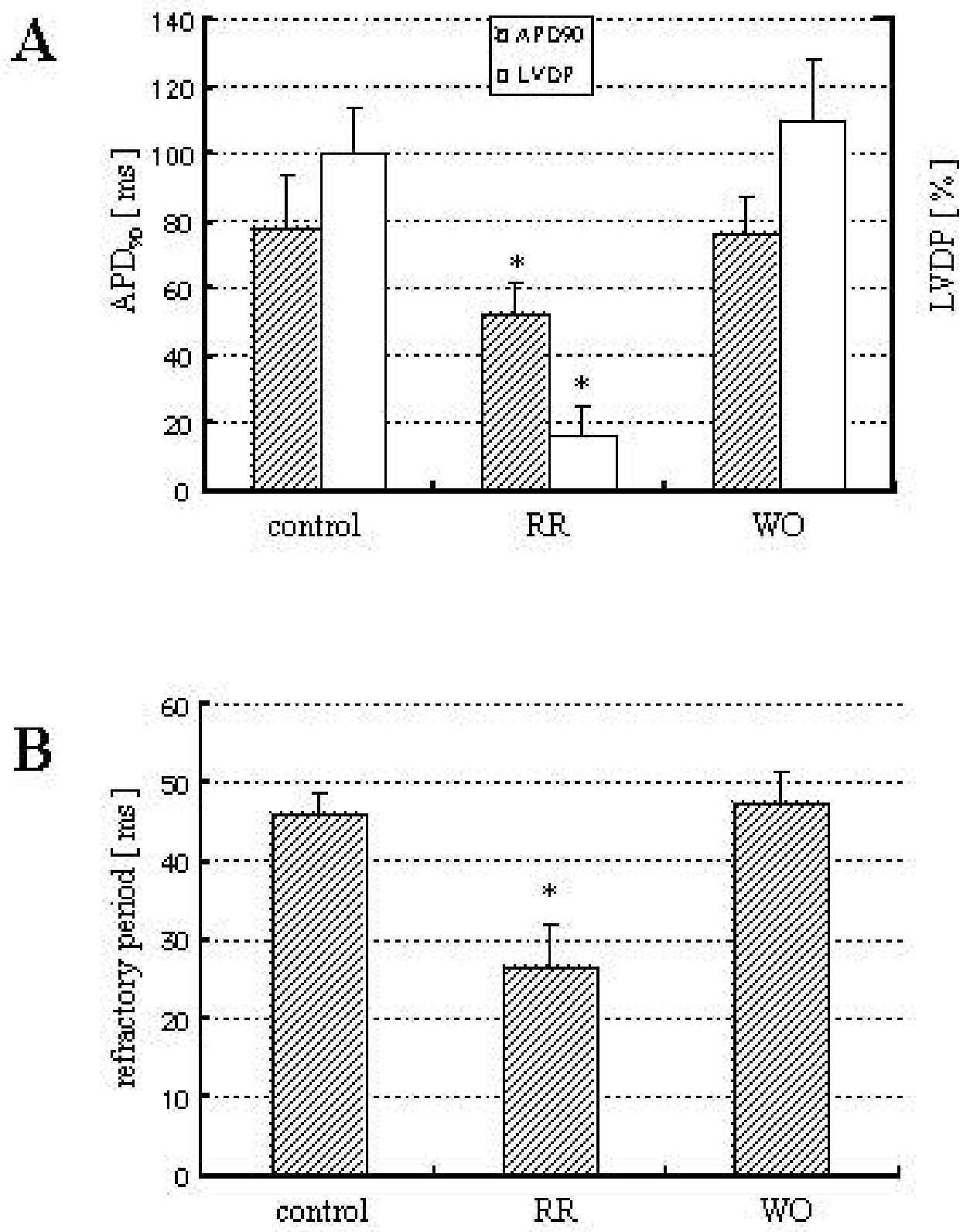


\section{A1}

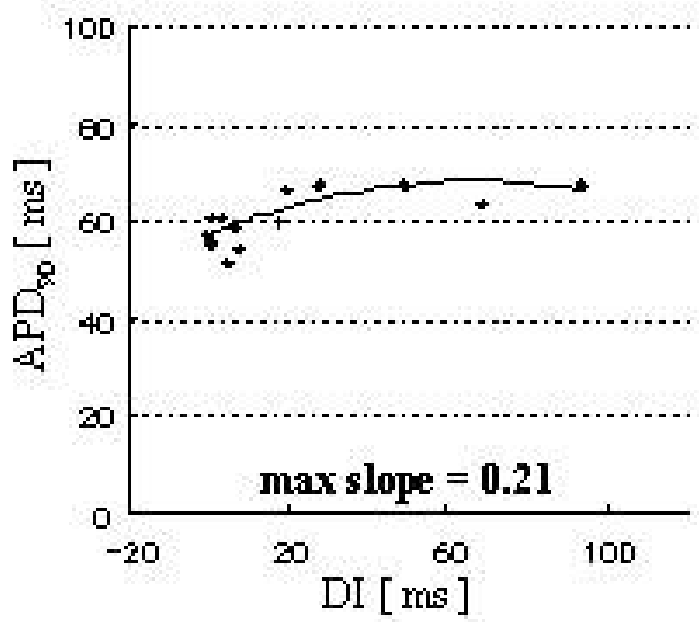

A2

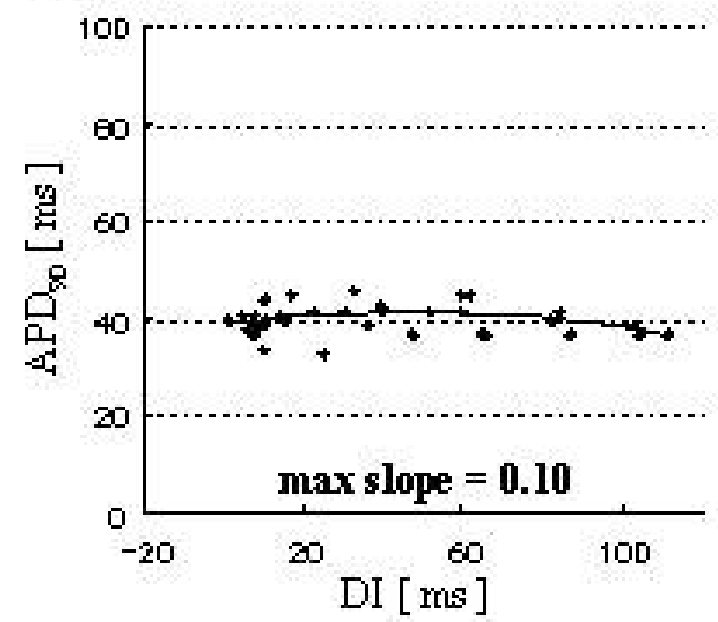

A3

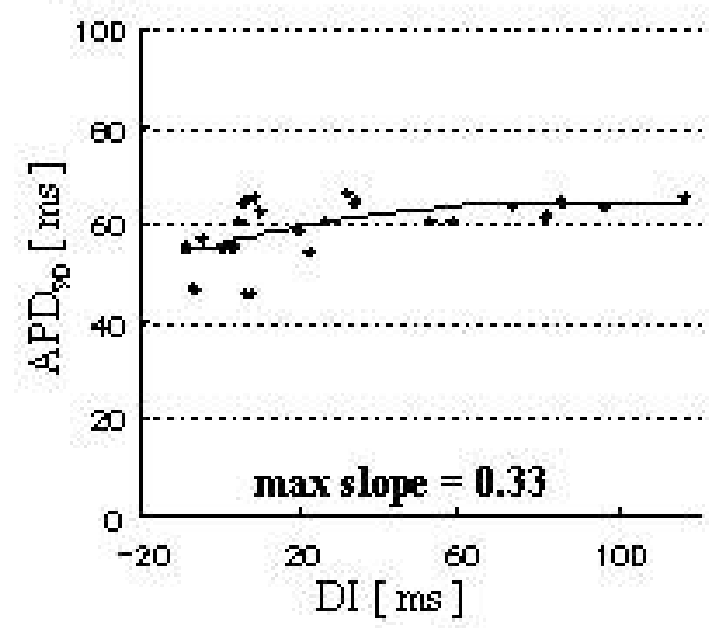

B

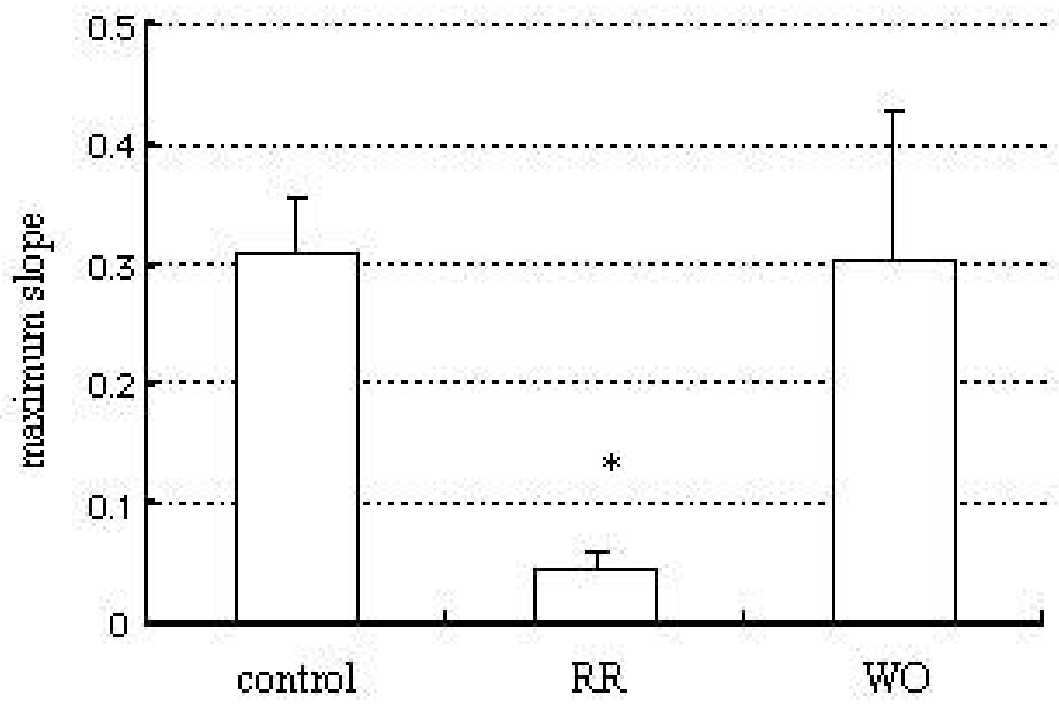



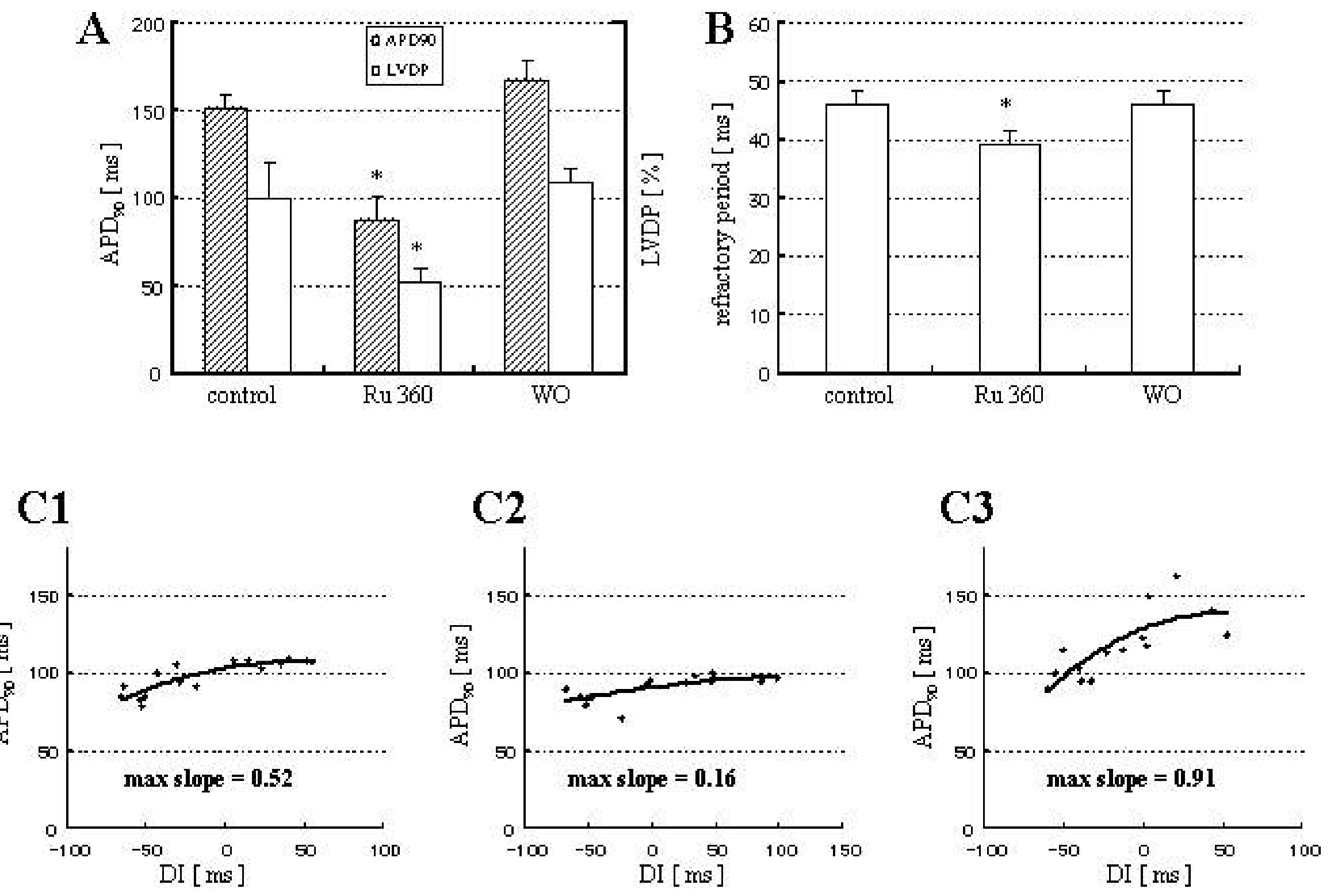

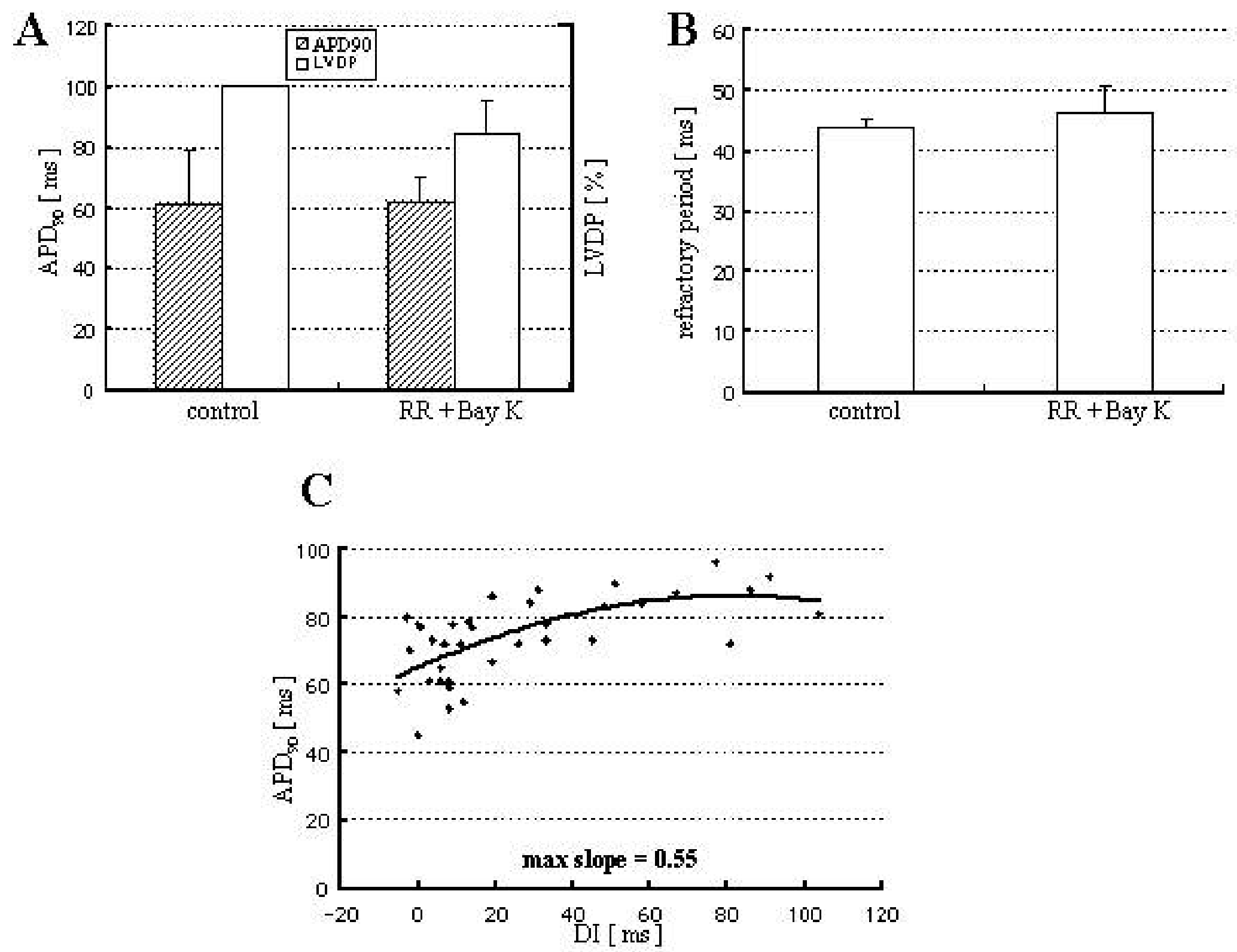


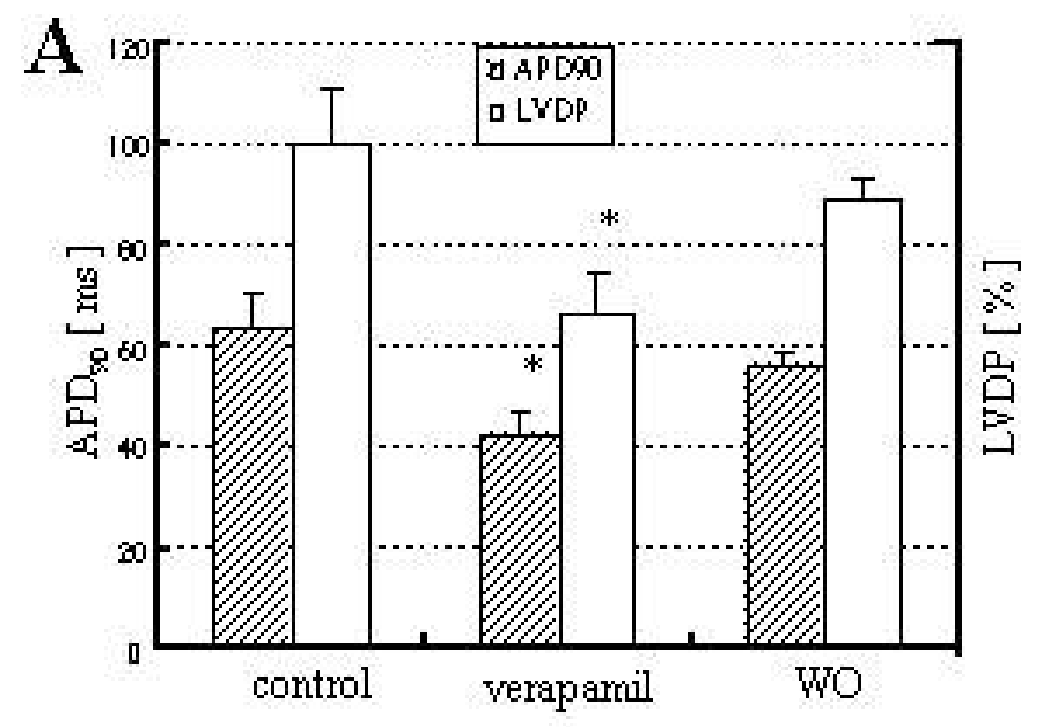

C1

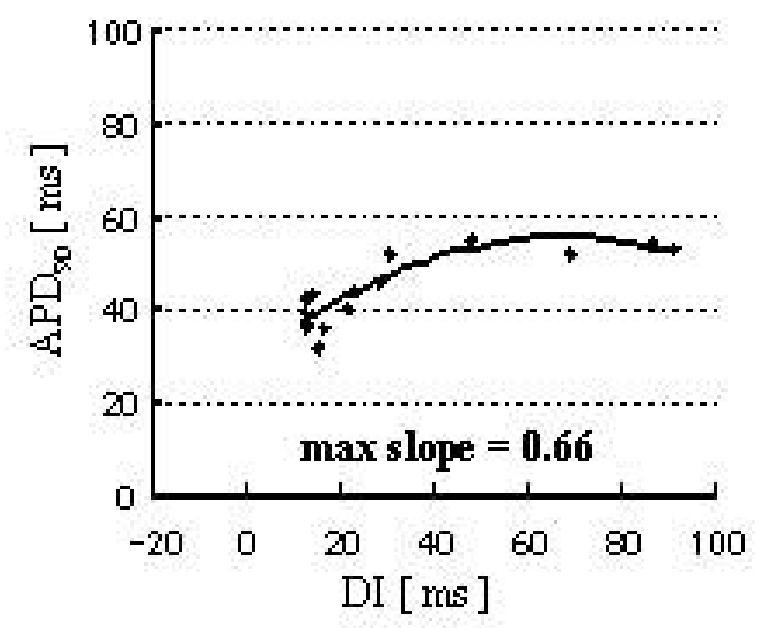

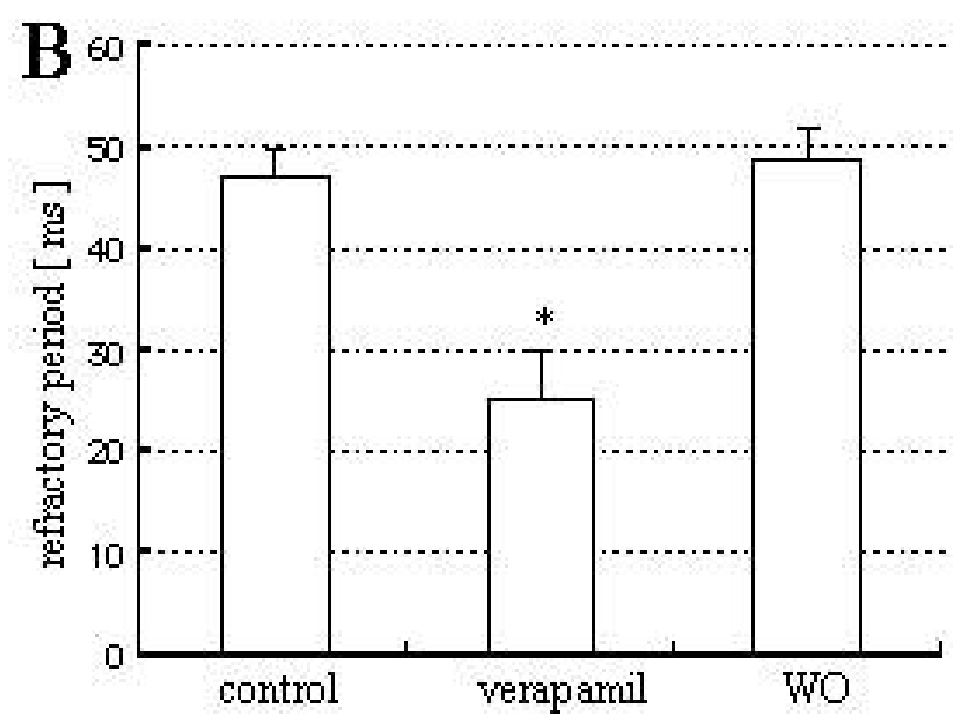

C3

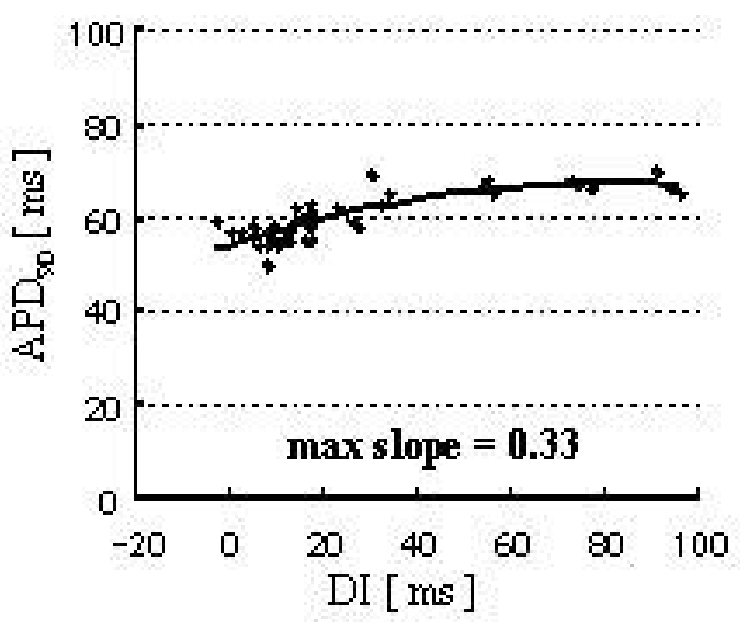

